CORRECTION

https://doi.org/10.1038/s41586-019-1107-5

\title{
Author Correction: Single-cell analysis of mixed-lineage states leading to a binary cell fate choice
}

Andre Olsson, Meenakshi Venkatasubramanian,

Virendra K. Chaudhri, Bruce J. Aronow, Nathan Salomonis, Harinder Singh \& H. Leighton Grimes

Correction to: Nature https://doi.org/10.1038/nature19348, published online 31 August 2016.

In this Letter, the first name of author Virendra K. Chaudhri was incorrectly spelled 'Viren'. In addition, author Meenakshi Venkatasubramanian should have also been associated with the affiliation 'Department of Electrical Engineering and Computer Science, University of Cincinnati, Cincinnati, Ohio 45221, USA', and authors Bruce J. Aronow, Nathan Salomonis, Harinder Singh and H. Leighton Grimes should all have also been associated with the affiliation 'Department of Pediatrics, University of Cincinnati College of Medicine, Cincinnati, Ohio 45229, USA'. The original Letter has not been corrected online. 